\title{
Image Saliency Prediction in Transformed Domain: A Deep Complex Neural Network Method
}

\author{
Lai Jiang, Zhe Wang, Mai Xu, ${ }^{*}$ Zulin Wang \\ Beihang University, Beijing 100191, China \\ *Corresponding Author: Mai Xu (maixu@buaa.edu.cn)
}

\begin{abstract}
The transformed domain fearures of images show effectiveness in distinguishing salient and non-salient regions. In this paper, we propose a novel deep complex neural network, named SalDCNN, to predict image saliency by learning features in both pixel and transformed domains. Before proposing Sal-DCNN, we analyze the saliency cues encoded in discrete Fourier transform (DFT) domain. Consequently, we have the following findings: 1) the phase spectrum encodes most saliency cues; 2) a certain pattern of the amplitude spectrum is important for saliency prediction; 3 ) the transformed domain spectrum is robust to noise and down-sampling for saliency prediction. According to these findings, we develop the structure of SalDCNN, including two main stages: the complex dense encoder and three-stream multi-domain decoder. Given the new SalDCNN structure, the saliency maps can be predicted under the supervision of ground-truth fixation maps in both pixel and transformed domains. Finally, the experimental results show that our Sal-DCNN method outperforms other 8 state-of-theart methods for image saliency prediction on 3 databases.
\end{abstract}

\section{Introduction}

Saliency prediction is a widely studied computer vision task, aiming to understand and predict human visual attention on images/videos. The last decade has witnessed the success of applying transformed domain methods in saliency prediction, since the transformed domain features has been verified to be effective in distinguishing salient and non-salient regions. In the beginning, (Hou and Zhang 2007) proposed predicting image saliency in discrete Fourier transform (DFT) domain, by subtracting the locally averaged amplitude spectrum from the original one. Afterwards, a number of transformed domain methods (Guo and Zhang 2010; Hou, Harel, and Koch 2012; Li et al. 2015; Leboran et al. 2017) were proposed for saliency prediction, mainly focusing on 2 aspects. 1) Transform on input channels, e.g., DFT (Hou and Zhang 2007), quaternion Fourier transform (QFT) (Guo and Zhang 2010) and discrete Cosine transform (DCT) (Hou, Harel, and Koch 2012). 2) Algorithms in the transformed domain, e.g., spectral residual (Hou and Zhang 2007), phase filtering (Li et al. 2015) and amplitude spectrum normalization (Guo and Zhang 2010). But all these methods rely on the hand-designed transformed

Copyright (c) 2019, Association for the Advancement of Artificial Intelligence (www.aaai.org). All rights reserved. domain features, which may be not well suitable for image saliency.

Most recently, instead of hand-designed features, deep neural network (DNN) based methods (Pan et al. 2017; Cornia et al. 2018; Wang and Shen 2018; Huang et al. 2015; Cornia et al. 2016; Jetley, Murray, and Vig 2016; Xu et al. 2018b) show the outstanding performance by end-to-end training for saliency prediction. In these methods, advanced DNN architectures, e.g., LSTM-based (Cornia et al. 2018), GAN-based (Pan et al. 2017) and multi-scale (Wang and Shen 2018) structures, were proposed to extract saliency cues from images. Besides, others focused on developing effective loss functions, e.g., Kullback CLeibler (KL) divergence based (Huang et al. 2015), location bias (Cornia et al. 2016) and Bhattacharyya distance (Jetley, Murray, and Vig 2016) loss. However, the existing DNNs only focus on pixel domain, ignoring the transformed domain features that highly contribute to saliency prediction ( $\mathrm{Li}$ et al. 2013).

Inspired by complex CNN (Trabelsi et al. 2017), this paper proposes a novel Sal-DCNN method, to predict image saliency by learning features at both pixel and transformed domains. We first investigate how saliency cues are encoded in DFT domain, along with the following findings. 1) The phase spectrum encodes most saliency cues; 2) a certain pattern of the amplitude spectrum is important for saliency prediction; 3 ) the transformed domain spectrum is robust to noise and down-sampling for saliency prediction. Following these findings, we propose an encoder-decoder structure for Sal-DCNN. Specifically, based on the developed complex components, a complex dense encoder is proposed in this paper to extract deep features considering both pixel and transformed domain information. Then, a three-stream multidomain decoder is proposed to predict not only saliency in pixel domain, but also the spectrums of phase and residual amplitude for saliency in the transformed domain. Finally, the saliency map of an image can be generated by fusing pixel and transformed domain saliency.

\section{Related work}

Recently, the transformed domain methods (Hou and Zhang 2007; Guo and Zhang 2010; Leboran et al. 2017) have been widely studied for saliency prediction. The main idea of these methods is that the salient objects and non-salient background can be easily distinguished in the transformed domain. Be- 
sides, the transformed domain methods can be seen as the approximation of saliency response in the primary visual cortex (V1 cells), considering biological processes such as orientation selectivity and lateral surround inhibition (Bian and Zhang 2008; Li et al. 2013).

The fundamental process of a transformed domain method is selecting an effective transformed domain, in which the visual saliency can be easily predicted. As a pioneering work in 2007, (Hou and Zhang 2007) applied DFT on images, and then extracted the spectral residual (SR) from DFT domain for saliency prediction. After that, a number of methods (Fang et al. 2012; Leboran et al. 2017; Li et al. 2015; Wang, Zhang, and $\mathrm{Li}$ 2016) were proposed to predict image/video saliency in DFT domain. For instance, DFT was extended to 3D in (Wang, Zhang, and Li 2016) for further considering temporal information in video saliency prediction. Inspired by DFT based methods, Guo (Guo and Zhang 2010) significantly advanced saliency prediction by applying QFT with the input of 4 feature channels. Afterwards, QFT was applied in many saliency prediction works ( $\mathrm{Li}$ et al. 2013). Besides, (Xu et al. 2017) has investigated that the saliency can be effectively predicted in HEVC domain (Li et al. 2017; Xu et al. 2018a).

The effective calculation in the transformed domain is also important, with the goal of popping out the salient regions while suppressing the non-salient regions. In the early time, (Hou and Zhang 2007) calculated the spectral residual of log amplitude spectrum, and then used inverse DFT (IDFT) to generate saliency maps. However, (Guo and Zhang 2010) found that it can achieve comparable performance when removing the amplitude spectrum. Most recently, advanced algorithms have been proposed in the transformed domain, focusing on both amplitude and phase spectrums (Fang et al. 2012; Li et al. 2013; Leboran et al. 2017; Li et al. 2015). Specifically, (Li et al. 2013) found that the repeated patterns in the background can lead to the spikes in the amplitude spectrum. As such, a specific filter was designed in the transformed domain to suppress the sharp spikes. Similarly, the phase filters were learned in ( $\mathrm{Li}$ et al. 2015) by minimizing Cosine distance between the phase spectrums of output and ground-truth saliency maps.

However, most of the above transformed domain methods are hand-designed, without automatically learning the transformed domain features from large-scale training data. On the other hand, DNNs (Huang et al. 2015; Cornia et al. 2016; Pan et al. 2017; Sun et al. 2017; Dodge and Karam 2018; Cornia et al. 2018; Wang and Shen 2018; Jiang et al. 2018) have been widely used in pixel domain to learn visual saliency on images. Specifically, to learn hierarchical saliency features, (Wang and Shen 2018) conducted a multi-level supervision in the convolutional (conv) layers with different receptive fields. Besides, (Huang et al. 2015) and (Cornia et al. 2016) focused on developing the suitable loss function of DNN for saliency prediction. In (Huang et al. 2015), the KL divergence based loss was verified to be efficient in training DNN for saliency prediction, while the location bias loss was first considered in (Cornia et al. 2016). Moveover, LSTM-based and GANbased DNNs were proposed in (Cornia et al. 2018) and (Pan et al. 2017) for image saliency.

Nevertheless, the existing DNNs all work in pixel domain, which have no access to leverage the transformed domain features for saliency prediction. In contrast, the complex structures have been verified to have a richer representation capacity (Mandic and Goh 2009). In this paper, we propose a novel Sal-DCNN, to take advantage of both transformed and pixel domain features for image saliency prediction.

\section{Transformed domain analysis}

As introduced above, image saliency can be effectively predicted in the transformed domain. Here, we conduct both qualitative and quantitative experiments over a widely used database MIT1003 (Judd et al. 2009) to investigate saliency cues encoded in the transformed domain, by analyzing and recovering the ground-truth fixation maps in DFT domain. For each fixation map $G$ (in the form of gray-scale heat map), DFT $(\mathcal{F})$ is applied to obtain phase spectrum $\mathcal{P}_{G}$ and amplitude spectrum $\mathcal{A}_{\boldsymbol{G}}$, denoted by

$$
\begin{aligned}
\mathcal{F}(\boldsymbol{G}(x, y)) & =\mathcal{A}_{\boldsymbol{G}}(u, v) \cdot e^{\imath \cdot \mathcal{P}_{\boldsymbol{G}}(u, v)} \\
& =\boldsymbol{\mathcal { R }}_{\boldsymbol{G}}(u, v)+\imath \cdot \mathcal{I}_{\boldsymbol{G}}(u, v),
\end{aligned}
$$

where $\mathcal{R}_{G}$ and $\mathcal{I}_{G}$ are the real and imaginary parts of DFT domain coefficients. Then, 3 findings are investigated as follows.

Finding 1. The phase spectrum encodes most saliency cues of a fixation map.

Since some transformed domain methods (Li et al. 2013; 2015) are based on amplitude and phase spectrums, it is worth finding out which spectrum contributes more for predicting saliency. To this end, given the fixation maps of 2 images ( $\boldsymbol{G}$ and $\boldsymbol{G}^{\prime}$ ), we exchange the amplitude spectrums $\left(\mathcal{A}_{\boldsymbol{G}}\right.$ and $\left.\mathcal{A}_{G^{\prime}}\right)$, and then apply IDFT $\left(\mathcal{F}^{-1}\right)$ to generate saliency maps. Similar operations are also conducted for the real parts of DFT domain coefficients $\left(\mathcal{R}_{\boldsymbol{G}}\right.$ and $\left.\mathcal{R}_{G^{\prime}}\right)$. Consequently, 4 sets of saliency maps are obtained as follows,

$$
\begin{array}{ll}
\text { - } \mathcal{A}_{G} \text { with } \mathcal{P}_{G^{\prime}}: & \mathcal{F}^{-1}\left(\mathcal{A}_{G}(u, v) \cdot e^{\imath \cdot \mathcal{P}_{G}^{\prime}(u, v)}\right), \\
\text { - } \mathcal{A}_{G^{\prime}} \text { with } \mathcal{P}_{G}: & \mathcal{F}^{-1}\left(\mathcal{A}_{G}^{\prime}(u, v) \cdot e^{\imath \cdot \mathcal{P}_{G}(u, v)}\right), \\
\text { - } \mathcal{R}_{G} \text { with } \mathcal{I}_{G^{\prime}}: & \mathcal{F}^{-1}\left(\mathcal{R}_{G}(u, v)+\imath \cdot \mathcal{I}_{G}^{\prime}(u, v)\right), \\
\text { - } \mathcal{R}_{G^{\prime}} \text { with } \mathcal{I}_{G}: & \mathcal{F}^{-1}\left(\mathcal{R}_{G}^{\prime}(u, v)+\imath \cdot \mathcal{I}_{G}(u, v)\right) .
\end{array}
$$

As we can see from Figure 1, the combination of $\mathcal{A}_{G^{\prime}}$ and $\mathcal{P}_{G}$ can well recover fixation map $G$. Similarly, the combination of $\mathcal{A}_{G}$ and $\mathcal{P}_{G^{\prime}}$ is able to effectively recover $G^{\prime}$. This indicates that the saliency cues are encoded in phase spectrum, rather than amplitude spectrum. We can also see from this figure that the real or imaginary part contributes to recovering visual saliency to some extent, but not as accurate as the phase based saliency maps. In Table 1, similar findings can be investigated from the quantitative results over MIT1003, by measuring correlation coefficient (CC) and KL divergence between recovered saliency maps and groundtruth fixation maps. Note that a smaller KL divergence means better performance. Therefore, the phase spectrum is able to encode most saliency cues of a fixation map.

Finding 2. A certain pattern of the amplitude spectrum is needed to recover a fixation map.

As found above, the phase spectrum is able to recover the fixation map, even when combined with amplitude spectrum 

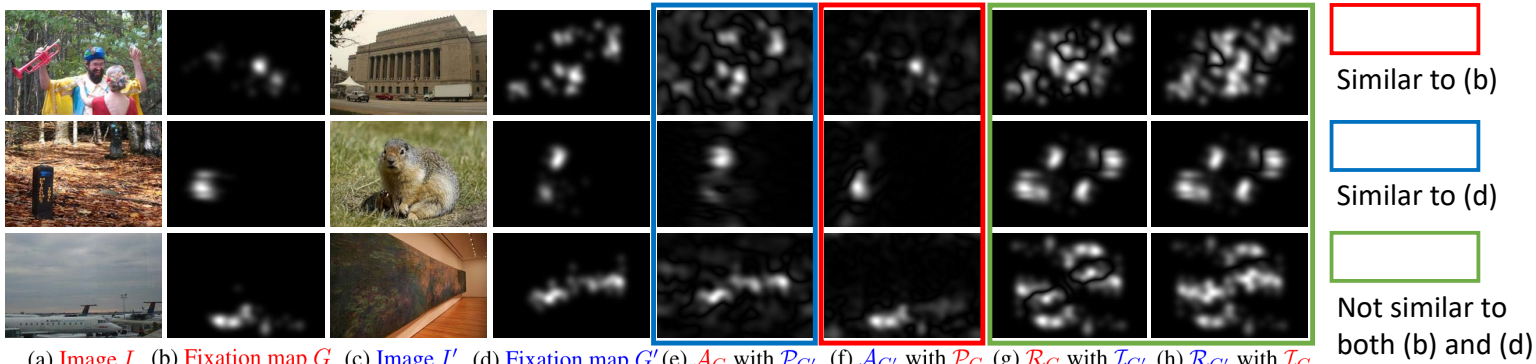

Figure 1: Original images (a, c), fixation maps (b, d) and their recovered saliency maps based on the combinations of $\mathcal{A}_{G}$ with $\mathcal{P}_{G^{\prime}}(\mathrm{e}), \mathcal{A}_{G^{\prime}}$ with $\mathcal{P}_{G}$ (f), $\mathcal{R}_{G}$ with $\mathcal{I}_{G^{\prime}}(\mathrm{g})$ and $\mathcal{R}_{G^{\prime}}$ with $\mathcal{I}_{G}$ (h). Note that red/blue color in the notations refers to the same source.

Table 1: Performance of saliency maps recovered for Finding 1\&2, obtained over MIT1003.

\begin{tabular}{|c|c|c|c|c|c|c|c|c|}
\hline & \multicolumn{4}{|c|}{ Recovered with different combinations (Finding 1) } & \multicolumn{4}{|c|}{ Recovered with replaced amplitude (Finding 2) } \\
\hline & $\mathcal{A}_{G}$ with $\mathcal{P}_{G^{\prime}}$ & $\mathcal{A}_{G^{\prime}}$ with $\mathcal{P}_{G}$ & $\mathcal{R}_{G}$ with $\mathcal{I}_{G^{\prime}}$ & $\mathcal{R}_{G^{\prime}}$ with $\mathcal{I}_{G}$ & Averaged amplitude & Image amplitude & Fixed value & WGN \\
\hline $\mathrm{CC}$ & 0.21 & 0.90 & 0.60 & 0.62 & 0.94 & 0.71 & 0.41 & 0.01 \\
\hline KL & 2.03 & 0.58 & 0.88 & 0.83 & 0.54 & 1.32 & 1.39 & 2.06 \\
\hline
\end{tabular}

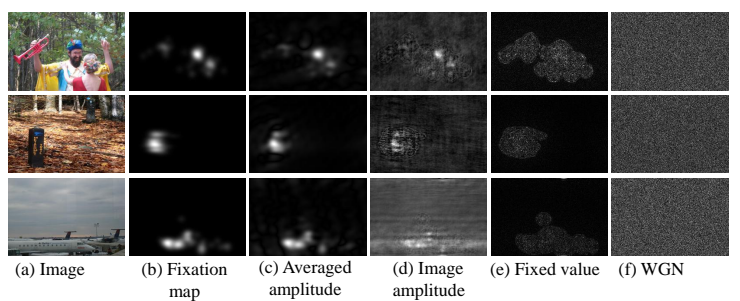

Figure 2: Original images (a), fixation maps (b) and saliency maps recovered from the replaced amplitude spectrums: the averaged amplitude of all fixation maps (c), the amplitude of original image (d), the amplitude with fixed value (e) and WGN (f).

Table 2: Consistency evaluation for 4 DFT domain spectrums/parts over MIT1003.

\begin{tabular}{ccccc}
\hline & Amplitude & Phase & Real & Imaginary \\
\hline $\mathrm{CC}$ & 0.93 & 0.03 & 0.64 & 0.08 \\
\hline
\end{tabular}

of another fixation map. It is worth investigating whether the amplitude spectrum is completely unnecessary for recovering a fixation map. To this end, assume that the mean and standard deviation (Std) of the amplitude spectrum are $m_{a}$ and $\sigma_{a}$ for each fixation map $\boldsymbol{G}$. Then, we replace the amplitude spectrum by 4 strategies: I) The averaged amplitude of all $N$ fixation maps in MIT1003, II) the amplitude of its original image $\boldsymbol{I}$, III) the amplitude with fixed value of $m_{a}$ and IV) the white Gassuian noise (WGN) with $m_{a}$ and $\sigma_{a}$. The corresponding saliency maps are then obtained as,

- Strategy I $\mathcal{F}^{-1}\left(\left(\frac{1}{N} \Sigma_{n=1}^{N} \mathcal{A}_{n}(u, v)\right) \cdot e^{\imath \cdot \mathcal{P}_{G}(u, v)}\right)$,

- Strategy II $\mathcal{F}^{-1}\left(\mathcal{A}_{\boldsymbol{I}}(u, v) \cdot e^{\imath \cdot \mathcal{P}_{\boldsymbol{G}}(u, v)}\right)$,

- Strategy III $\mathcal{F}^{-1}\left(m_{a} \cdot e^{\imath \cdot \mathcal{P}_{G}(u, v)}\right)$,
- Strategy IV $\mathcal{F}^{-1}\left(\operatorname{AWGN}\left(m_{a}, \sigma_{a}\right) \cdot e^{\imath \cdot \mathcal{P}_{G}(u, v)}\right)$.

Note that $\operatorname{AWGN}(\cdot, \cdot)$ is the 2D WGN map. The performance of those recovered saliency maps can be found in Figure 2 and Table 1, in which Strategies II)-IV) apparently fail to recover the fixation maps. This implies that the amplitude spectrum with a certain pattern is necessary for recovering a fixation map. Moreover, we further measure the $\mathrm{CC}$ values of 4 single spectrums/parts and their averaged spectrums/parts over MIT1003. As shown in Table 2, in comparison with phase, real and imaginary, the amplitude spectrums of different fixation maps are highly consistent. This indicates that the amplitude spectrums of fixation maps have a general pattern, encoding little saliency cues. In summary, we find that although the amplitude spectrum encodes little saliency cues, a certain pattern is still needed for amplitude spectrum to recover a fixation map.

Finding 3. The phase spectrum is robust to noise and down-sampling for saliency prediction.

In our experiment, we find that the phase spectrums tend to be random. Therefore, it is interesting to evaluate the antinoise capability of phase spectrum. Here, we add zero-mean WGN with different Std $\left(\{1,0.7,0.5,0.3,0.1,0.01\} \sigma_{p}\right)$ to each phase spectrum of fixation map $G$. Then, we test whether it can still recover the fixation map. Note that $\sigma_{p}$ is the Std of the original phase spectrum. The qualitative and quantitative results are shown in Figures 3 and 4, respectively. The horizontal axis of Figure 4 also lists the corresponding peak signal to noise ratio (PSNR), for each noise-added phase spectrum. According to Figures 3 and 4, the phase spectrum is capable of recovering the fixation map $(\mathrm{CC}=0.94$, $\mathrm{KL}=0.80$ ), even added by WGN with $0.3 \sigma_{p}$ Std (PSNR $=23.35 d B$ ). This verifies the phase spectrums has a good anti-noise capability for predicting image saliency.

Furthermore, we also evaluate the sensitivity of phase spectrum at different resolutions for recovering the fixation map. 


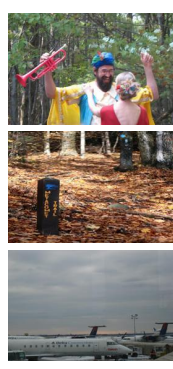

(a) Image

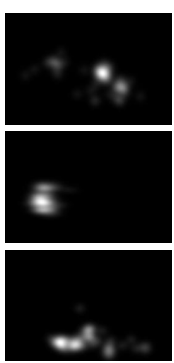

(b) Fixation map

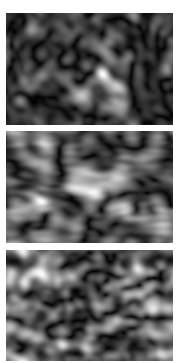

(c) $1 \sigma_{\mathrm{g}}^{p} \mathrm{WGN}$

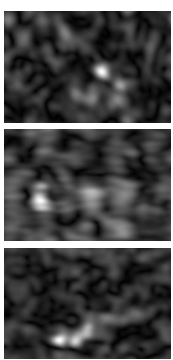

(d) $0.7 \sigma_{\mathrm{g}}^{p} \mathrm{WGN}$

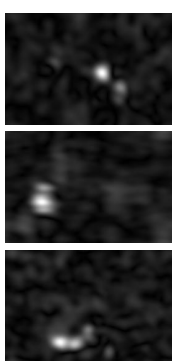

(e) $0.5 \sigma_{\mathrm{g}}^{p} \mathrm{WGN}$

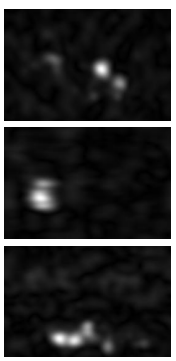

(f) $0.3 \sigma_{\mathrm{g}}^{p} \mathrm{WGN}$

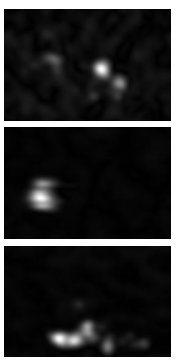

$\begin{array}{ll}\text { (g) } 0.1 \sigma_{\mathrm{g}}^{p} \mathrm{WGN} & \text { (h) } 0.01 \sigma_{\mathrm{g}}^{p} \mathrm{WGN}\end{array}$

Figure 3: Original images (a), fixation maps (b) and saliency maps recovered from noise-added phase spectrums (c)-(h).

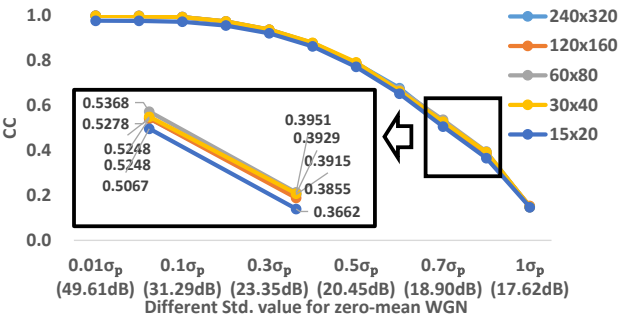

(a) $\mathrm{CC}$ values

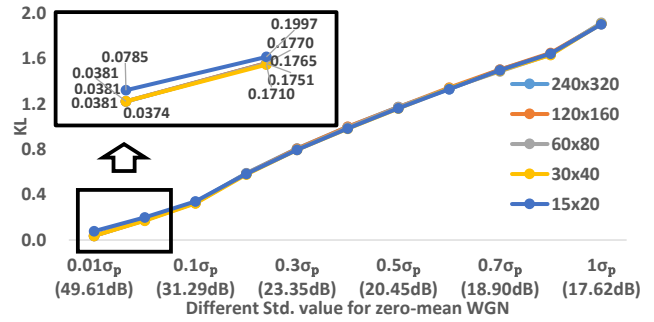

(b) KL divergences

Figure 4: Performance of saliency maps recovered from our experiments in Findings 3 over MIT1003, in the terms of CC (a) and KL (b). For each sub-figure, the horizontal axis indicates the phase spectrums added by different WGN. Note that the corresponding PSNR of each WGN is listed in brackets. Besides, the curves with different colors refer to the phase spectrums with different resolutions.

To this end, the phase spectrum of each fixation map is down sampled to $240 \times 320,120 \times 160,60 \times 80,30 \times 40$ and $15 \times 20$. Then, we evaluate the performance of the recovered saliency maps. Surprisingly, we can see from Figure 4 that the resolution of phase spectrum has little effect on recovering the fixation map. This finding is important for designing the phase-based loss function. In other words, we can reduce the dimension of ground-truth phase spectrum to improve the generalization ability of our learning algorithm. It is worth mentioning that above results can be found in amplitude spectrum.

\section{Proposed method}

The architecture of Sal-DCNN is shown in Figure 5. Inspired by the above findings, we develop Sal-DCNN considering both pixel and transformed information for saliency prediction. Basically, some complex components are designed in Sal-DCNN, in order to utilize the effective saliency cues of the transformed domain. Based on these complex components, a complex dense encoder is proposed to extract the deep complex features, from the RGB channels of the input image and their corresponding DFT coefficients. Then, given the deep complex features, a three-stream multi-domain decoder is developed to generate pixel domain saliency map, as well as the predicted spectrums of phase and amplitude. Consequently, the final saliency map can be obtained by fusing pixel and transformed domain saliency.

\subsection{Complex components}

In the following, we briefly introduce the complex components in our Sal-DCNN. Following the idea of (Hirose and Yoshida 2012), we use real-valued representations to model the basic components of complex CNN. As such, the proposed complex CNN can be easily implemented in the modern deep learning platforms. Taking the conv layer as an example, we can re-formulate the complex convolution operation by the real-valued convolution $(*)$ as follows,

$$
\left[\begin{array}{c}
\boldsymbol{s}_{r} \\
\boldsymbol{s}_{i}
\end{array}\right]=\left[\begin{array}{cc}
\boldsymbol{W}_{r} & -\boldsymbol{W}_{i} \\
\boldsymbol{W}_{i} & \boldsymbol{W}_{r}
\end{array}\right] *\left[\begin{array}{c}
\boldsymbol{z}_{r} \\
\boldsymbol{z}_{i}
\end{array}\right]+\left[\begin{array}{c}
\boldsymbol{\beta}_{r} \\
\boldsymbol{\beta}_{i}
\end{array}\right],
$$

where $r$ and $i$ represent the real and imaginary parts of the corresponding kernel weights $(\boldsymbol{W})$, biases $(\boldsymbol{\beta})$, input $(\boldsymbol{z})$ and output $(s)$ tensors. Similarly, in our method, the complex batch normalization (BN) and pooling layers are also represented by real-valued operations as in (Trabelsi et al. 2017). Additionally, we propose some new basic components, i.e., the squared-leakage rectified linear unit (ReLU) and channelwise polynomial projection, to make improvement on the existing complex activation layer and projection layer, respectively.

Squared-leakage ReLU. Different from the $\mathbb{C R e L U}$ and $z$ ReLU in (Trabelsi et al. 2017), we develop a squaredleakage ReLU to overcome the problems of losing phase information $(\mathbb{C R e L U})$ and sparse activation $(z \mathrm{ReLU})$. The 


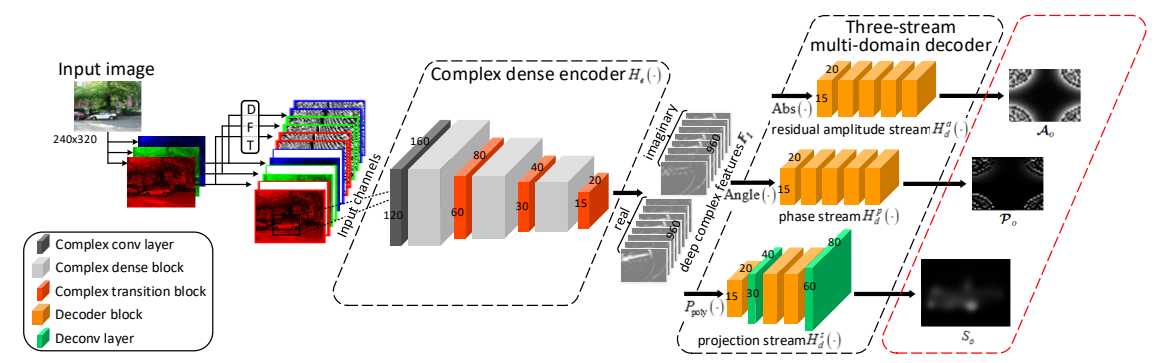

(a) Framework of Sal-DCNN

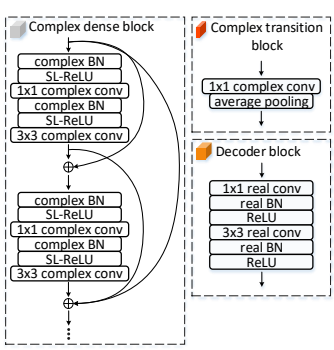

(b) Details of each block

Figure 5: Architecture of Sal-DCNN for image saliency prediction, including structures of complex dense encoder and threestream multi-domain decoder. Note that the channel numbers in the encoder rely on the hyper-parameters of growth rate and compression factor in the complex dense block and transition block. In the decoder stage, the channel number is summarized to be a half after each conv/deconv layer.

function for the squared-leakage ReLU is

$$
\mathrm{SL}-\operatorname{ReLU}(c)=\left\{\begin{array}{ll}
c & \text { if } \theta_{c} \in\left[0, \frac{\pi}{2}\right) \\
\alpha c & \text { if } \theta_{c} \in\left[\frac{\pi}{2}, \pi\right) \\
\alpha c & \text { if } \theta_{c} \in\left[\pi, \frac{3 \pi}{2}\right) \\
\alpha^{2} c & \text { if } \theta_{c} \in\left[\frac{3 \pi}{2}, 2 \pi\right)
\end{array},\right.
$$

where $c$ is the complex number needed to be activated; $\theta_{c}$ is the phase of $c$; and $\alpha$ is the leakage coefficient.

Channel-wise polynomial projection. The aim of projection layer is mapping the complex number into real number. Typically, the projection is simply conducted by computing the magnitude of complex number (Guberman 2016). In our method, we develop a channel-wise polynomial projection $P_{\text {poly }}$ to learn the optimal projection as follows,

$P_{\text {poly }}\left(\boldsymbol{z}^{c}\right)=\omega_{1}^{c} \cdot\left(\boldsymbol{z}_{r}^{c}\right)^{2}+\omega_{2}^{c} \cdot\left(\boldsymbol{z}_{i}^{c}\right)^{2}+\omega_{3}^{c} \boldsymbol{z}_{r}^{c} \boldsymbol{z}_{i}^{c}+\omega_{4}^{c} \boldsymbol{z}_{r}^{c}+\omega_{5}^{c} \boldsymbol{z}_{i}^{c}+\omega_{6}^{c}$,

where $z^{c}$ is the $c$-th channel of complex input, and $\left\{\omega_{i}\right\}_{i=1}^{6}$ are the second-order polynomial coefficients.

\subsection{Encoder and decoder structures}

Complex dense encoder. Based on the complex components, a complex dense encoder is proposed to encode the saliency cues in both pixel and transformed domain. Following (Huang et al. 2017), we develop complex dense block and complex transition block in our encoder structures. The details about these 2 blocks are shown in Figure 5-(b). For each input image $\boldsymbol{I}$, we first concatenate the RGB channels and their corresponding DFT coefficients ${ }^{1}$, as the input to the complex dense encoder. Note that each RGB channel is also represented as a complex number by setting its imaginary part to zero. Before concatenating, the DFT coefficients are normalized to have a similar scale as the RGB channels. Then, the input channels are fed into the complex dense encoder, which includes 1 complex conv layer, 3 complex dense

\footnotetext{
${ }^{1}$ Since DFT is a global operation over the whole image, the DFT coefficients can be seen as the features with receptive field of input resolution. As such, this concatenation can also act as a multi-scale integration.
}

blocks and 3 complex transition blocks, as shown in Figure 5. It is worth noting that each complex conv layer in the encoder is followed by a complex BN, a linear and a squared-leakage $R e L U$ layers. Consequently, the deep complex features $\boldsymbol{F}_{\boldsymbol{I}}$ can be extracted as

$$
\boldsymbol{F}_{\boldsymbol{I}}=H_{e}\left(\boldsymbol{I} ; \boldsymbol{W}_{e}\right),
$$

where $H_{e}(\cdot)$ indicates our complex dense encoder with learnable weights $\boldsymbol{W}_{e}$ in all complex blocks and layers.

In summary, there are 3 advantages for our complex dense encoder. 1) The input of the encoder contains both pixel and transformed domain information; 2) The complex components of the encoder make it possible to extract deep complex features considering the transformed domain. 3) The advanced dense and transition blocks can strengthen information flow only with a small number of parameters.

Three-stream multi-domain decoder. As introduced in Finding 1, the transformed domain information, especially the phase spectrum, contributes to predicting image saliency. Thus, a three-stream multi-domain decoder is proposed to generate not only the predicted saliency map in pixel domain, but also the predicted spectrums of phase and amplitude in the transformed domain (see Figure 5). Specifically, according to Finding 2, we propose a residual learning scheme in the amplitude stream of decoder. That is, instead of of predicting the ground-truth of amplitude spectrum, we predict the residual between the general pattern and ground-truth. As introduced in Finding 2, this general pattern $\mathcal{A}_{t}$ is obtained by averaging all ground-truth amplitude spectrums $\left(\mathcal{A}_{n}\right)$ in training set $\mathbb{T}$ with the total number of $N$, as follows

$$
\mathcal{A}_{t}=\frac{1}{N} \sum_{n \in \mathbb{T}} \mathcal{A}_{n} \text {. }
$$

Given the deep complex features $\boldsymbol{F}_{\boldsymbol{I}}$ extracted from the complex dense encoder, we can obtain the pixel domain saliency map $\boldsymbol{S}_{o}$, predicted phase spectrum $\mathcal{P}_{o}$ and predicted residual amplitude spectrum $\mathcal{A}_{o}$ by

$$
\begin{aligned}
\boldsymbol{S}_{o} & =H_{d}^{s}\left(P_{\text {poly }}\left(\boldsymbol{F}_{\boldsymbol{I}}\right) ; \boldsymbol{W}_{d}^{s}\right), \\
\mathcal{P}_{o} & =H_{d}^{p}\left(\operatorname{Angle}\left(\boldsymbol{F}_{\boldsymbol{I}}\right) ; \boldsymbol{W}_{d}^{p}\right), \\
\mathcal{A}_{o} & =H_{d}^{a}\left(\operatorname{Abs}\left(\boldsymbol{F}_{\boldsymbol{I}}\right) ; \boldsymbol{W}_{d}^{a}\right) .
\end{aligned}
$$




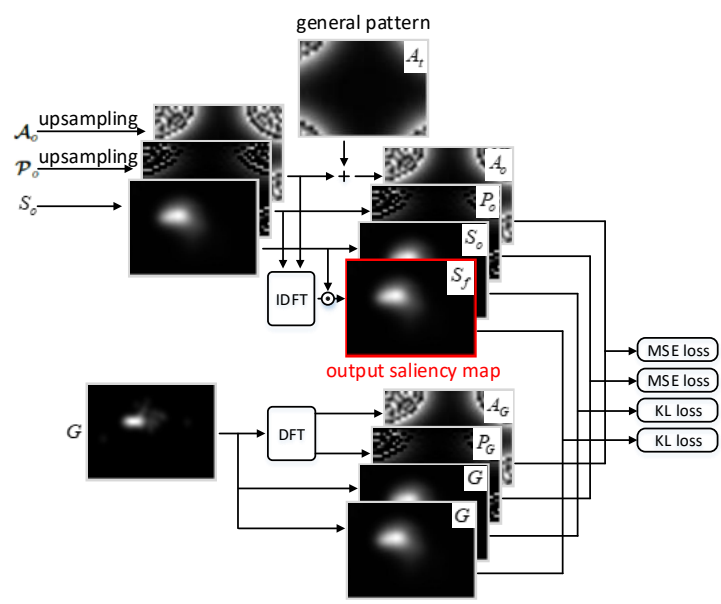

Figure 6: Structure for final saliency map generation and the loss functions in Sal-DCNN.

In (7), $P_{\text {poly }}(\cdot)$ is the proposed channel-wise polynomial projection in (4), while $\operatorname{Angle}(\cdot)$ and $\operatorname{Abs}(\cdot)$ are the operations to calculate the phase and magnitude of $\boldsymbol{F}_{\boldsymbol{I}}$. Besides, $\boldsymbol{W}_{d}^{s}$, $\boldsymbol{W}_{d}^{p}$ and $\boldsymbol{W}_{d}^{a}$ indicate the learnable weights in 3 decoder streams, i.e., projection stream $H_{d}^{s}(\cdot)$, phase stream $H_{d}^{p}(\cdot)$ and residual amplitude stream $H_{d}^{a}(\cdot)$. For the detailed structures of the decoder, see Figure 5.

The decoder part has 3 advantages. 1) Transformed domain saliency can be learned through the phase and residual amplitude steams of the decoder. 2) The residual learning scheme improves the efficiency on predicting the groundtruth amplitude spectrum. 3) Both pixel and transformed domain saliency are decoded for saliency prediction.

\subsection{Loss function}

Given pixel domain saliency map $\boldsymbol{S}_{o}$, predicted phase spectrum $\mathcal{P}_{o}$ and predicted residual amplitude spectrum $\mathcal{A}_{o}$, we can obtain final saliency map $\boldsymbol{S}_{f}$ by fusing pixel and transformed domain saliency, as follows

$$
\boldsymbol{S}_{f}=\frac{\omega_{f}^{2}}{\omega_{f}^{2}+1} \boldsymbol{S}_{o}+\frac{1}{\omega_{f}^{2}+1} \mathcal{F}^{-1}\left(\left(\mathcal{A}_{o}+\mathcal{A}_{t}\right) \cdot e^{\imath \cdot \mathcal{P}_{o}}\right)
$$

where $\omega_{f}$ is the learnable weight for integrating pixel and transformed domain saliency. The fusing process is also shown in Figure 6.

To train Sal-DCNN, predicted phase spectrum $\mathcal{P}_{o}$ and residual amplitude spectrum $\mathcal{A}_{o}$ are supervised, via computing mean-square error (MSE) between the predicted and ground-truth spectrums $\left(\mathcal{P}_{G}\right.$ and $\left.\mathcal{A}_{G}\right)$. Meanwhile, regarded as the probability distributions of attention, pixel domain saliency map $\boldsymbol{S}_{o}$ and final saliency map $\boldsymbol{S}_{f}$ are supervised by ground-truth fixation map $\boldsymbol{G}$ through KL divergence minimization. Finally, the overall loss of Sal-DCNN can be defined by

$$
\begin{aligned}
\operatorname{LOSS} & =\underbrace{\operatorname{KL}\left(\boldsymbol{S}_{f}, \boldsymbol{G}\right)}_{\text {final loss }}+\underbrace{\lambda_{o} \operatorname{KL}\left(\boldsymbol{S}_{o}, \boldsymbol{G}\right)}_{\text {projection loss }} \\
& +\underbrace{\lambda_{p} \frac{\operatorname{MSE}\left(\mathcal{P}_{o}, \mathcal{P}_{\boldsymbol{G}}\right)}{R_{p}}}_{\text {phase loss }}+\underbrace{\lambda_{a} \frac{\operatorname{MSE}\left(\mathcal{A}_{o}+\mathcal{A}_{t}, \mathcal{A}_{\boldsymbol{G}}\right)}{R_{a}}}_{\text {amplitude loss }},
\end{aligned}
$$

where $\lambda_{o}, \lambda_{p}$ and $\lambda_{a}$ are hyper-parameters for controlling the weights for projection loss, phase loss and amplitude loss. Besides, $R_{p}$ and $R_{a}$ are the factors to re-scale MSE based loss to the similar range of KL based loss. The details about the loss function is also shown in Figure 6.

Table 3: Implementation details and hyper-parameters.

\begin{tabular}{ll}
\hline Leakage coefficient in (3) & 0.1 \\
Conv layer numbers of 3 dense blocks & $6,12,32$ \\
Growth rate of complex dense block & 48 \\
Compression factor of complex transition block & 0.5 \\
Loss weights $\lambda_{o}, \lambda_{p}$ and $\lambda_{a}$ in (9) & $0.5,0.5,0.1$ \\
Re-scaling factors $R_{p}$ and $R_{a}$ in (9) & 10,20 \\
Initial learning rate & $1 \times 10^{-5}$ \\
Training iterations & $\sim 1.4 \times 10^{5}$ \\
Weight decay & $5 \times 10^{-6}$ \\
\hline
\end{tabular}

\section{Experiment}

\subsection{Settings.}

In this section, the experimental results are presented to verify the effectiveness of our Sal-DCNN method. In our experiments, the Sal-DCNN model is trained over the training set (10,000 images) of (Jiang et al. 2015). The model training is based on the stochastic gradient descent algorithm with the Adam optimizer. The whole training process takes around 15 hours in a computer with $3.4 \mathrm{GHz}$ CPU, a GTX 1080 GPU, and 32G RAM. Here, the hyper-parameters of our method are tuned over 5,000 images in the validation set of (Jiang et al. 2015). The implementation details and tuned hyper-parameters are listed in Table 3. Then, our Sal-DCNN method is tested on 3 widely used large-scale image saliency databases, i.e., MIT1003 (Judd et al. 2009), CAT2000 (Borji and Itti 2015) and DUT (Yang et al. 2013). We randomly split these 3 databases into training and test sets at a ratio of $4: 1$, so the numbers of test images for MIT1003, CAT2000 and DUT are 201, 400 and 1034. Note that we do not evaluate the performance of Sal-DCNN on the test set of (Jiang et al. 2015), as it is not available online.

\subsection{Comparison Results.}

In our experiments, we compare the performance of image saliency prediction between our Sal-DCNN and other stateof-the-art methods, including SR (Hou and Zhang 2007), PQFT (Guo and Zhang 2010), BMS (Zhang and Sclaroff 2016), SALICON (Huang et al. 2015), ML-Net (Cornia et al. 2016), SalGAN (Pan et al. 2017), DVA (Wang and Shen 2018) and SAM (Cornia et al. 2018). Here, we use the codes of these methods available online for image saliency prediction in our performance comparison. Then, 4 metrics are 
Table 4: Averaged results for saliency prediction by our and 8 other methods over 3 databases.

\begin{tabular}{|c|cccc|cccc|cccc|}
\hline & \multicolumn{3}{|c|}{ MIT1003 (201 test images) } & \multicolumn{3}{c|}{ CAT2000 (400 test images) } & \multicolumn{3}{c|}{ DUT (1,034 test images) } \\
\cline { 2 - 27 } & AUC & NSS & CC & KL & AUC & NSS & CC & KL & AUC & NSS & CC & KL \\
\hline SALICON & 0.82 & 1.28 & 0.42 & 1.61 & 0.77 & 0.99 & 0.39 & 1.17 & 0.85 & 2.27 & 0.48 & 1.24 \\
DVA & 0.86 & $\mathbf{2 . 1 9}$ & $\mathbf{0 . 6 6}$ & $\mathbf{0 . 8 7}$ & 0.81 & 1.50 & 0.56 & 0.84 & 0.91 & $\mathbf{3 . 1 1}$ & 0.67 & 0.88 \\
SalGAN & 0.87 & 2.05 & 0.65 & 0.96 & 0.81 & 1.47 & 0.56 & 0.97 & 0.91 & 2.80 & 0.68 & 0.90 \\
ML-Net & 0.84 & 2.01 & 0.61 & 1.01 & 0.79 & 1.37 & 0.51 & 0.99 & 0.88 & 2.87 & 0.61 & 1.15 \\
SAM & 0.87 & 2.19 & 0.61 & 1.30 & 0.84 & 1.74 & 0.63 & 1.12 & 0.91 & 2.96 & 0.67 & 1.07 \\
BMS & 0.77 & 1.15 & 0.37 & 1.43 & 0.78 & 1.20 & 0.46 & 1.07 & 0.83 & 1.76 & 0.42 & 1.40 \\
PQFT $^{*}$ & 0.70 & 0.78 & 0.25 & 1.67 & 0.75 & 0.98 & 0.37 & 1.18 & 0.77 & 1.26 & 0.33 & 1.53 \\
SR $^{*}$ & 0.70 & 0.80 & 0.25 & 1.69 & 0.72 & 0.87 & 0.32 & 6.05 & 0.67 & 0.70 & 0.15 & 3.54 \\
\hline \hline Sal-DCNN & $\mathbf{0 . 8 7}$ & 2.10 & 0.62 & 0.89 & $\mathbf{0 . 8 6}$ & $\mathbf{2 . 0 3}$ & $\mathbf{0 . 7 9}$ & $\mathbf{0 . 6 3}$ & $\mathbf{0 . 9 2}$ & 3.07 & $\mathbf{0 . 7 6}$ & $\mathbf{0 . 5 5}$ \\
Sal-DCNN-PP & 0.86 & 1.98 & 0.61 & 0.93 & 0.86 & 2.00 & 0.77 & 0.65 & 0.92 & 3.06 & 0.75 & 0.57 \\
Sal-DCNN-P & 0.86 & 1.97 & 0.60 & 0.98 & 0.86 & 1.99 & 0.76 & 0.74 & 0.92 & 3.05 & 0.75 & 0.60 \\
Sal-DenseNet & 0.85 & 1.95 & 0.59 & 1.04 & 0.86 & 1.95 & 0.74 & 0.97 & 0.91 & 3.03 & 0.74 & 0.63 \\
\hline
\end{tabular}

* Transformed domain methods.

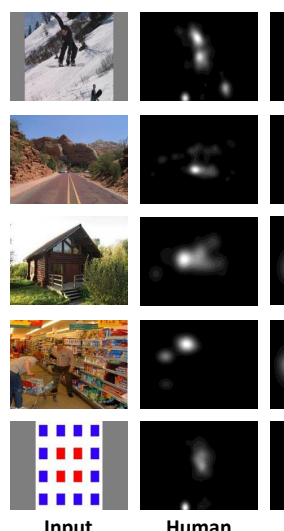

Input
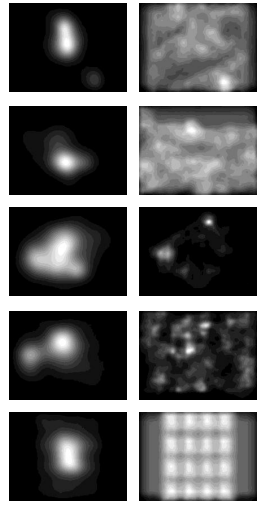

SALICON
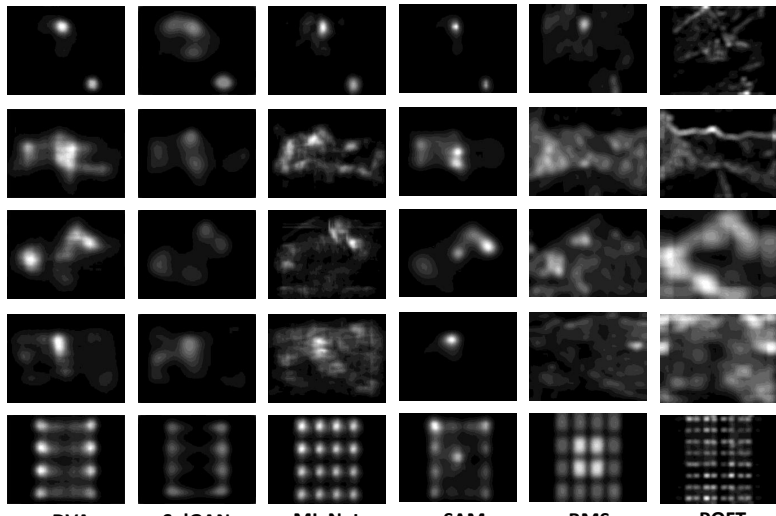

ML-Net

SAM
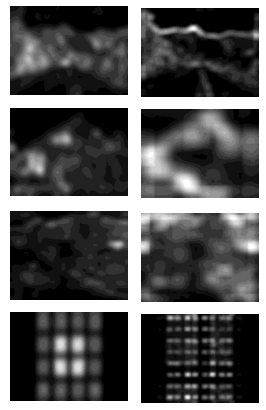

BMS

PQFT
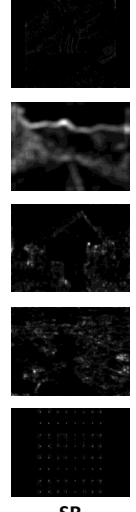

Figure 7: Saliency maps of 5 randomly selected images from MIT1003, CAT2000 and DUT. The maps were yielded by our and 8 other methods as well the ground-truth human fixations.

applied to measure the performance of saliency prediction: the area under the receiver operating characteristic curve (AUC), normalized scanpath saliency (NSS), CC, and KL divergence. Note that the larger value of AUC, NSS or CC indicates more accurate prediction of saliency, while a smaller KL divergence means better saliency prediction. As shown in Table 4, our method outperforms other state-of-the-art methods over MIT1003, CAT2000 and DUT in terms of most metrics. In particular, the gains of our Sal-DCNN method over SAM are 0.02 in AUC, 0.29 in NSS, 0.16 in CC, 0.49 in KL divergence, when tested on CAT2000. Note that SAM performs the best among all compared methods. In addition to the above quantitative results, Figure 7 shows that our SalDCNN method is capable of well locating the salient regions, which are closer to ground-truth fixations maps than other methods.

\subsection{Ablation Results.}

We further conduct the ablation experiments to analyze the contribution of each component proposed in our method. Specifically, we train the following models independently.
1) Sal-DenseNet: the real-valued dense encoder followed by the projection decoder stream; 2) Sal-DCNN-P: the complex dense encoder followed by the projection decoder stream; 3 ) Sal-DCNN-PP: the complex dense encoder followed by the projection and phase decoder streams (the amplitude spectrum is fixed by general pattern $\mathcal{A}_{t}$ ). As such, Sal-DenseNet is a complete real-valued $\mathrm{CNN}$ with the similar structure to Sal-DCNN, while Sal-DCNN-P only decodes the pixel domain saliency. We can see from Table 4 that compared with Sal-DenseNet, the transformed domain information in Sal-DCNN-P can reduce KL divergence by 0.23 over the images of CAT2000. Besides, the transformed domain saliency learned from the phase stream and residual amplitude stream has 0.09 and $0.02 \mathrm{KL}$ reduction over CAT2000, respectively. It is also worth mentioning that the squared-leakage ReLU and channel-wise polynomial projection, as the developed complex components in our Sal-DNN method, can also bring 0.01 and 0.03 reduction in KL divergence. The above results validate the effectiveness of the proposed components in our Sal-DCNN method. 


\section{Conclusion}

This paper has proposed the Sal-DCNN method for image saliency prediction, in which both pixel and transformed domain features are learned from the large-scale training images. Specifically, we found that a fixation map is mainly determined by the phase spectrum of its DFT coefficients. We also found that a certain pattern of the amplitude spectrum is necessary to recover the fixation map. The additional findings show that the phase spectrum of the DFT domain fixation maps is robust to noise and down-sampling. Inspired by our findings, we developed a new deep complex CNN structure for Sal-DCNN. The experimental results showed the proposed Sal-DCNN method can advance state-of-the-art image saliency prediction.

Acknowledgment This work was supported by the National Nature Science Foundation of China under Grants 61573037 and 61876013, and by the Fok Ying Tung Education Foundation under Grant 151061.

\section{References}

Bian, P., and Zhang, L. 2008. Biological plausibility of spectral domain approach for spatiotemporal visual saliency. In International conference on neural information processing, 251-258. Springer.

Borji, A., and Itti, L. 2015. Cat2000: A large scale fixation dataset for boosting saliency research. CVPR.

Cornia, M.; Baraldi, L.; Serra, G.; and Cucchiara, R. 2016. A Deep Multi-Level Network for Saliency Prediction. In International Conference on Pattern Recognition (ICPR).

Cornia, M.; Baraldi, L.; Serra, G.; and Cucchiara, R. 2018. Predicting Human Eye Fixations via an LSTM-based Saliency Attentive Model. IEEE Transactions on Image Processing.

Dodge, S. F., and Karam, L. J. 2018. Visual saliency prediction using a mixture of deep neural networks. IEEE Transactions on Image Processing 27(8):4080-4090.

Fang, Y.; Lin, W.; Lee, B.-S.; Lau, C.-T.; Chen, Z.; and Lin, C.W. 2012. Bottom-up saliency detection model based on human visual sensitivity and amplitude spectrum. IEEE Transactions on Multimedia 14(1):187-198.

Guberman, N. 2016. On complex valued convolutional neural networks. arXiv preprint arXiv:1602.09046.

Guo, C., and Zhang, L. 2010. A novel multiresolution spatiotemporal saliency detection model and its applications in image and video compression. IEEE transactions on image processing 19(1):185198.

Hirose, A., and Yoshida, S. 2012. Generalization characteristics of complex-valued feedforward neural networks in relation to signal coherence. IEEE Transactions on Neural Networks and learning systems 23(4):541-551.

Hou, X., and Zhang, L. 2007. Saliency detection: A spectral residual approach. In Computer Vision and Pattern Recognition, 2007. CVPR'07. IEEE Conference on, 1-8. IEEE.

Hou, X.; Harel, J.; and Koch, C. 2012. Image signature: Highlighting sparse salient regions. IEEE transactions on pattern analysis and machine intelligence 34(1):194-201.

Huang, X.; Shen, C.; Boix, X.; and Zhao, Q. 2015. Salicon: Reducing the semantic gap in saliency prediction by adapting deep neural networks. In ICCV, 262-270.

Huang, G.; Liu, Z.; Van Der Maaten, L.; and Weinberger, K. Q. 2017. Densely connected convolutional networks. In CVPR.
Jetley, S.; Murray, N.; and Vig, E. 2016. End-to-end saliency mapping via probability distribution prediction. In Proceedings of the IEEE Conference on Computer Vision and Pattern Recognition, 5753-5761.

Jiang, M.; Huang, S.; Duan, J.; and Zhao, Q. 2015. Salicon: Saliency in context. In $C V P R$.

Jiang, L.; Xu, M.; Liu, T.; Qiao, M.; and Wang, Z. 2018. Deepvs: A deep learning based video saliency prediction approach. In Computer Vision-ECCV 2018. Springer.

Judd, T.; Ehinger, K.; Durand, F.; and Torralba, A. 2009. Learning to predict where humans look. In Computer Vision, 2009 IEEE 12th international conference on, 2106-2113. IEEE.

Leboran, V.; Garcia-Diaz, A.; Fdez-Vidal, X. R.; and Pardo, X. M. 2017. Dynamic whitening saliency. IEEE transactions on pattern analysis and machine intelligence 39(5):893-907.

Li, J.; Levine, M. D.; An, X.; Xu, X.; and He, H. 2013. Visual saliency based on scale-space analysis in the frequency domain. IEEE transactions on pattern analysis and machine intelligence 35(4):996-1010.

Li, J.; Duan, L.-Y.; Chen, X.; Huang, T.; and Tian, Y. 2015. Finding the secret of image saliency in the frequency domain. IEEE transactions on pattern analysis and machine intelligence 37(12):24282440.

Li, S.; Xu, M.; Wang, Z.; and Sun, X. 2017. Optimal bit allocation for ctu level rate control in hevc. IEEE Transactions on Circuits and Systems for Video Technology 27(11).

Mandic, D. P., and Goh, V. S. L. 2009. Complex valued nonlinear adaptive filters: noncircularity, widely linear and neural models, volume 59. John Wiley \& Sons.

Pan, J.; Sayrol, E.; Giro-i Nieto, X.; Ferrer, C. C.; Torres, J.; McGuinness, K.; and O'Connor, N. E. 2017. Salgan: Visual saliency prediction with adversarial networks. In CVPR Scene Understanding Workshop (SUNw).

Sun, X.; Huang, Z.; Yin, H.; and Shen, H. T. 2017. An integrated model for effective saliency prediction. In $A A A I$.

Trabelsi, C.; Bilaniuk, O.; Zhang, Y.; Serdyuk, D.; Subramanian, S.; Santos, J. F.; Mehri, S.; Rostamzadeh, N.; Bengio, Y.; and Pal, C. J. 2017. Deep complex networks. arXiv preprint arXiv:1705.09792.

Wang, W., and Shen, J. 2018. Deep visual attention prediction. IEEE Transactions on Image Processing 27(5).

Wang, Y.; Zhang, Q.; and Li, B. 2016. Efficient unsupervised abnormal crowd activity detection based on a spatiotemporal saliency detector. In WACV, 1-9. IEEE.

Xu, M.; Jiang, L.; Sun, X.; Ye, Z.; and Wang, Z. 2017. Learning to detect video saliency with hevc features. IEEE Transactions on Image Processing 26(1).

Xu, M.; Li, T.; Wang, Z.; Deng, X.; Yang, R.; and Guan, Z. 2018a. Reducing complexity of hevc: A deep learning approach. IEEE Transactions on Image Processing.

Xu, M.; Song, Y.; Wang, J.; Qiao, M.; Huo, L.; and Wang, Z. 2018b. Predicting head movement in panoramic video: A deep reinforcement learning approach. IEEE transactions on pattern analysis and machine intelligence.

Yang, C.; Zhang, L.; Lu, H.; Ruan, X.; and Yang, M.-H. 2013. Saliency detection via graph-based manifold ranking. In $C V P R$.

Zhang, J., and Sclaroff, S. 2016. Exploiting surroundedness for saliency detection: a boolean map approach. IEEE transactions on pattern analysis and machine intelligence 38(5):889-902. 